\title{
BMJ Open Health outcomes, utility and costs of returning incidental results from genomic sequencing in a Canadian cancer population: protocol for a mixed- methods randomised controlled trial
}

\author{
Salma Shickh, ${ }^{1,2}$ Marc Clausen, ${ }^{2}$ Chloe Mighton, ${ }^{1,2}$ Mariana Gutierrez Salazar, ${ }^{2,3}$ \\ Kathleen-Rose Zakoor, ${ }^{3}$ Rita Kodida, ${ }^{2}$ Emma Reble, ${ }^{2}$ Christine Elser, ${ }^{4,5}$ \\ Andrea Eisen, ${ }^{6}$ Seema Panchal, ${ }^{5}$ Melyssa Aronson, ${ }^{7}$ Tracy Graham, ${ }^{6}$ \\ Susan Randall Armel, ${ }^{8}$ Chantal F Morel, ${ }^{4,9}$ Ramzi Fattouh, ${ }^{10,11}$ Emily Glogowski, ${ }^{12}$ \\ Kasmintan A Schrader, ${ }^{13,14}$ Jada G Hamilton, ${ }^{15}$ Kenneth Offit, ${ }^{15}$ Mark Robson, ${ }^{15,16}$ \\ June C Carroll, ${ }^{17,18}$ Wanrudee Isaranuwatchai, ${ }^{1,19}$ Raymond H Kim, ${ }^{4,20}$ \\ Jordan Lerner-Ellis, ${ }^{3,11}$ Kevin E Thorpe, ${ }^{21,22}$ Andreas Laupacis, ${ }^{4,23}$ \\ Yvonne Bombard, ${ }^{1,2}$ On behalf of the Incidental Genomics Study Team
}

To cite: Shickh S, Clausen M, Mighton C, et al. Health outcomes, utility and costs of returning incidental results from genomic sequencing in a Canadian cancer population: protocol for a mixed-methods randomised controlled trial. BMJ Open 2019;9:e031092. doi:10.1136/ bmjopen-2019-031092

- Prepublication history and additional material for this paper are available online. To view these files, please visit the journal online (http://dx.doi. org/10.1136/bmjopen-2019031092).

Received 15 April 2019 Revised 12 July 2019 Accepted 19 July 2019

Check for updates

(C) Author(s) (or their employer(s)) 2019. Re-use permitted under CC BY-NC. No commercial re-use. See rights and permissions. Published by BMJ.

For numbered affiliations see end of article.

Correspondence to Dr Yvonne Bombard; yvonne.bombard@utoronto.ca

\section{ABSTRACT}

Introduction Genomic sequencing has rapidly transitioned into clinical practice, improving diagnosis and treatment options for patients with hereditary disorders. However, large-scale implementation of genomic sequencing faces challenges, especially with regard to the return of incidental results, which refer to genetic variants uncovered during testing that are unrelated to the primary disease under investigation, but of potential clinical significance. High-quality evidence evaluating health outcomes and costs of receiving incidental results is critical for the adoption of genomic sequencing into clinical care and to understand the unintended consequences of adoption of genomic sequencing. We aim to evaluate the health outcomes and costs of receiving incidental results for patients undergoing genomic sequencing.

Methods and analysis We will compare health outcomes and costs of receiving, versus not receiving, incidental results for adult patients with cancer undergoing genomic sequencing in a mixed-methods randomised controlled trial. Two hundred and sixty patients who have previously undergone first or second-tier genetic testing for cancer and received uninformative results will be recruited from familial cancer clinics in Toronto, Ontario. Participants in both arms will receive cancer-related results. Participants in the intervention arm have the option to receive incidental results. Our primary outcome is psychological distress at 2 weeks following return of results. Secondary outcomes include behavioural consequences, clinical and personal utility assessed over the 12 months after results are returned and health service use and costs at 12 months and 5 years. A subset of participants and providers will complete qualitative interviews about utility of incidental results.

Ethics and dissemination This study has been approved by Clinical Trials Ontario Streamlined Research Ethics

\section{Strengths and limitations of this study}

- This randomised controlled trial (RCT) will provide high-quality evidence on the outcomes and costs of receiving incidental results (IR) from genomic sequencing, providing critical evidence given the increasing use of genomic sequencing and identification of IR.

- Most studies thus far have evaluated only the return of medically actionable and carrier results, however this RCT will offer patients a wide range of IR options, representing the most comprehensive assessment that we know of to date.

- Our study will include qualitative interviews with participants and providers, enabling an in-depth understanding of the personal and clinical utility of IR.

- All participants will have the option to receive IR at the end of the study period; however, this limits our ability to compare the long-term economic costs of receiving versus not receiving IR.

- We will recruit relatives and providers of participants to explore family spillover effects, care consequences and costs associated with $I R$, representing a unique opportunity to gain novel and comprehensive insights into the cascading effects of IR on health services and the family system.

Review System that provides ethical review and oversight for multiple sites participating in the same clinical trial in Ontario.

Results from the trial will be shared through stakeholder workshops, national and international conferences, and peer-reviewed journals.

Trial registration number NCT03597165. 


\section{INTRODUCTION}

As the cost of sequencing technologies continues to decrease, the use of genomic sequencing (GS; including both exome and genome sequencing) is increasing in clinical practice. ${ }^{2}$ One critical ethical and policy challenge is that GS often identifies incidental results (IR; also known as secondary findings), which refer to gene variants that are unrelated to the primary disease but that may or may not be clinically useful. American guidelines recommend that individuals undergoing GS in a clinical setting be offered the option to receive medically actionable results associated with at least 59 genes. ${ }^{3}$ Other guidelines recommend returning results from these and additional genes. ${ }^{45}$ European and Canadian guidelines are more conservative, and recommend that GS be targeted to genomic regions associated with the primary indication for testing, to reduce IR. However, IR may be returned, with patients receiving IR based on their preferences identified during pretest counselling. ${ }^{6-8}$ In the UK, investigators are encouraged to return IR that are clinically relevant, actionable and associated with moderate or severe life-threatening diseases. ${ }^{9}$ Thus, there is general consensus that patients undergoing clinical GS should have the option to receive actionable IR. ${ }^{347}$

Despite current recommendations to return only actionable results, patients and the public seem interested in receiving a broader range of IR which includes results associated with untreatable conditions, carrier status results and to a lesser degree, variants of unknown significance (VUS). ${ }^{10-13}$ They also express interest in IR conferring risk of cancer, neurological and other disorders. ${ }^{10-15}$ These preferences appear to be consistent across both hypothetical scenarios and real-world settings ${ }^{1012}$ and have been found across other areas of medicine. For example, $60 \%-90 \%$ of research participants indicate a preference for receiving incidental findings from magnetic resonance imaging (MRI). ${ }^{16} 17$

Motivators for learning various types of IR include a desire to learn personal health information and prepare for the future. ${ }^{14}$ Some commentators suggest that patient preferences should be considered in determining which results to return, regardless of whether they meet an actionability threshold. ${ }^{13} 18$ The literature indicates that the definition of a medically actionable result is subjective, and in some cases, clinicians and patients may perceive a result that is not medically actionable by guidelines to have some actionability and seek medical care. ${ }^{319-21}$ Given the varying interpretations of medical actionability, some argue for a more comprehensive approach to actionability and that in addition to clinical utility, personal utility should also be considered in shaping guidelines for the return of genomic results. ${ }^{13} 18$

To prepare for the anticipated large-scale implementation of GS and mainstream genomic medicine into clinical practice, the UK government recently completed the 100000 genomes project to sequence 100000 genomes. ${ }^{22}$ Using these data, researchers will develop methods to streamline the diagnostic process, educate professionals, and importantly, establish frameworks to return IR. ${ }^{922}$

IR provide an opportunity for disease risk reduction, and it is evident that patients are enthusiastic about receiving these results. However, there is little research evaluating actual health outcomes and economic consequences associated with receiving different categories of IR based on patient selections.

\section{STUDY AIMS}

\section{Hypotheses and aims}

We aim to evaluate the consequences of receiving IR on psychological distress, risk-reducing behaviour, clinical and personal utility in the 12 months following the return of results, as well as health service use and costs at 12 months and 5 years after results are returned. We hypothesise that, in comparison to patients receiving only primary results, patients receiving IR will report higher levels of short-term psychological distress but more risk-reducing behaviours to treat, screen or reduce incidentally identified disease risks, and higher levels of clinical and personal utility, thereby potentially improving health outcomes. Additionally, we hypothesise that health service utilisation and costs will be higher for patients receiving IR for short term (1 year) and longer term (5 years).

\section{METHODS AND ANALYSIS \\ Study design}

We will use a randomised controlled trial (RCT) to assess whether participants receiving IR and primary GS results will report higher levels of psychological distress, more risk-reducing behaviours, clinical and personal utility, health service use and costs compared with participants receiving primary results alone. We will use standardised measures administered over the course of 1 year (figure 1 and table 1). This protocol follows guidance from the Standard Protocol Items: Recommendations for Interventional Trials 2013 statement (online supplementary file 1). ${ }^{23} 24$

\section{Study setting}

The main study site is St. Michael's Hospital (SMH) in Toronto, Ontario, Canada, where all data collection activities will be coordinated and data will be stored. Participants will be recruited from hereditary cancer clinics at the Mount Sinai Hospital, Sunnybrook Health Sciences Centre, and Princess Margaret Cancer Centre located in Toronto. Recruitment began in July 2018 and is expected to end by September 2020.

\section{Population}

We selected patients with cancer for our study population as they represent a significant proportion of patients that may receive IR. Patients with cancer often undergo tumour DNA sequencing to identify prognostic biomarkers and to target treatments, ${ }^{25-27}$ which is often 


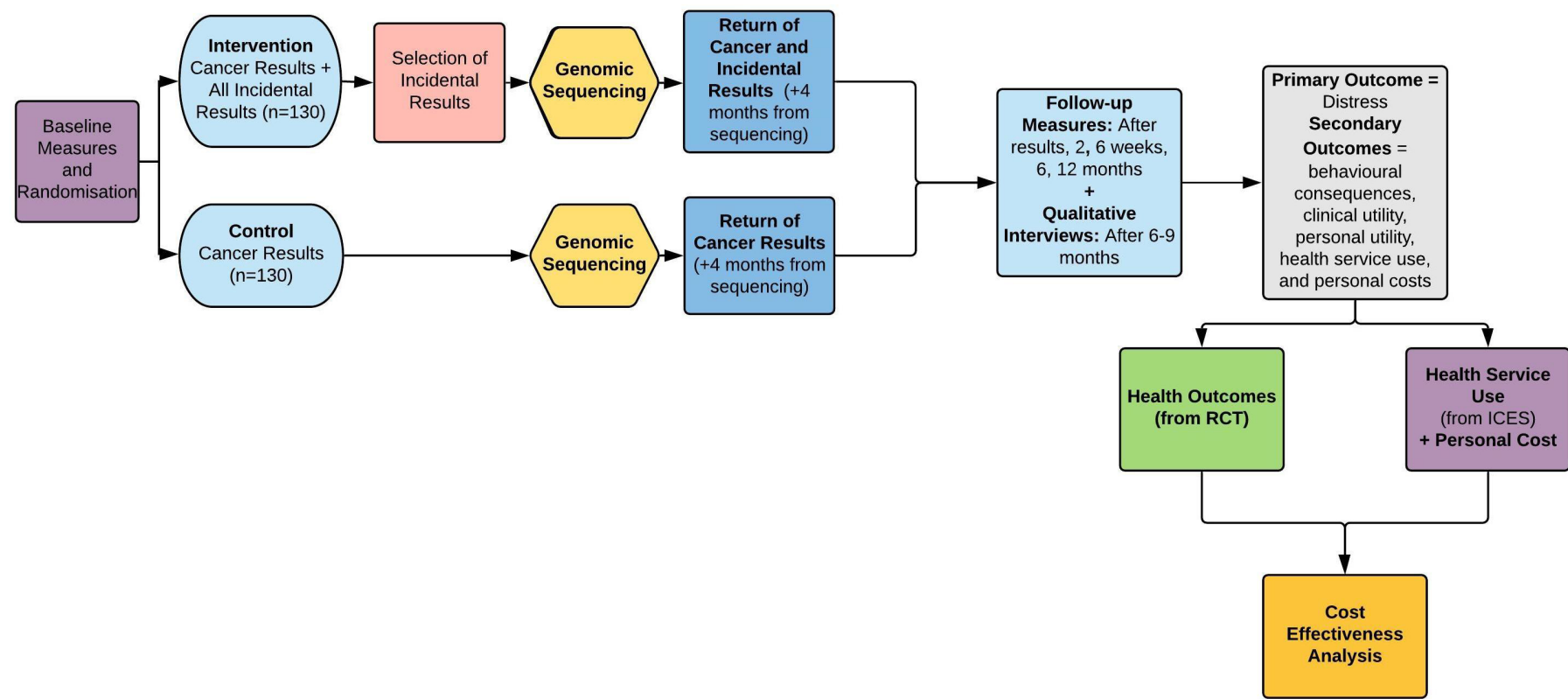

Figure 1 Study flow. ICES, Institute for Clinical and Evaluative Sciences; RCT, randomised controlled trial.

times accompanied by germline sequencing on normal control tissue. Germline GS also may offer increased sensitivity over classic genetic tests, resulting in improved diagnosis and decreased time-consuming and costly diagnostic cascades. ${ }^{28}$ However, germline sequencing may also lead to identification of IR. Therefore, patients with cancer represent a large population that may receive IR from GS.

\section{Inclusion and exclusion criteria}

We designed the eligibility criteria to reflect future clinical eligibility for GS.

\section{Inclusion criteria}

- Personal and family history of cancer or polyposis suggestive of a hereditary cancer syndrome.

- Previous uninformative genetic test results (negative or (VUS)).

$\checkmark \geq 18$ years of age.

- Speak and read English.

\section{Exclusion criteria}

- Received a positive or clinically significant (actionable) genetic result in a cancer gene (eg, BRCA1 pathogenic variant).

- Received previous GS.

- Patient or partner is pregnant or planning to become pregnant. This is to avoid any stress related to carrier results. If a participant or their partner becomes pregnant over the study period, they will not be excluded.

- Recurrent or metastatic cancer (stage 4), since IR could be perceived as burdensome to this population due to ongoing health challenges ${ }^{29}{ }^{30}$ or if they are in active cancer treatment.

- If they or relatives participated in the decision aid (DA) usability study or DA RCT that preceded this RCT. $^{1231}$

\section{Study arms}

Patients in the intervention and control arms will be offered GS for their cancer history.

\section{Intervention}

Participants in the intervention arm will receive cancer findings and be given the option to learn IR from up to five categories of results (figure 2). ${ }^{12}$ These categories were developed based on the binning model proposed by Berg $e t a l .^{32}$ The categories are organised by actionability, disease type and inheritance and have been tested for face validity and comprehension. ${ }^{12}$ We designed an online interactive DA called the 'Genomics ADvISER' ( www.genomicsadviser.com) ${ }^{12} 31$ that incorporates the binning framework and will guide intervention participants in their selection of IR (figure 3). The Genomics ADvISER has been evaluated and found to be acceptable, intuitive to use, and provides sufficient information for patients to reach an informed, value-congruent decision. ${ }^{12}$ Its effectiveness in reducing decisional conflict and improving knowledge for patients selecting IR was evaluated in a separate RCT. ${ }^{31}$

We will report pathogenic or likely pathogenic variants for IR [variants expected to be disease causing, as per the American College of Medical Genetics and Genomics (ACMG) guidelines]. ${ }^{33}$ For cancer-related results, we will report VUS results in addition to pathogenic and likely pathogenic variants, given that VUS may be reclassified and become relevant for participants' primary indication. ${ }^{33} 34$

\section{Control}

Participants in the control arm will only learn cancer-related GS results (primary indication) and will not receive IR. To account for recruitment bias, participants in the control arm will be able to obtain IR at the conclusion of the study. 


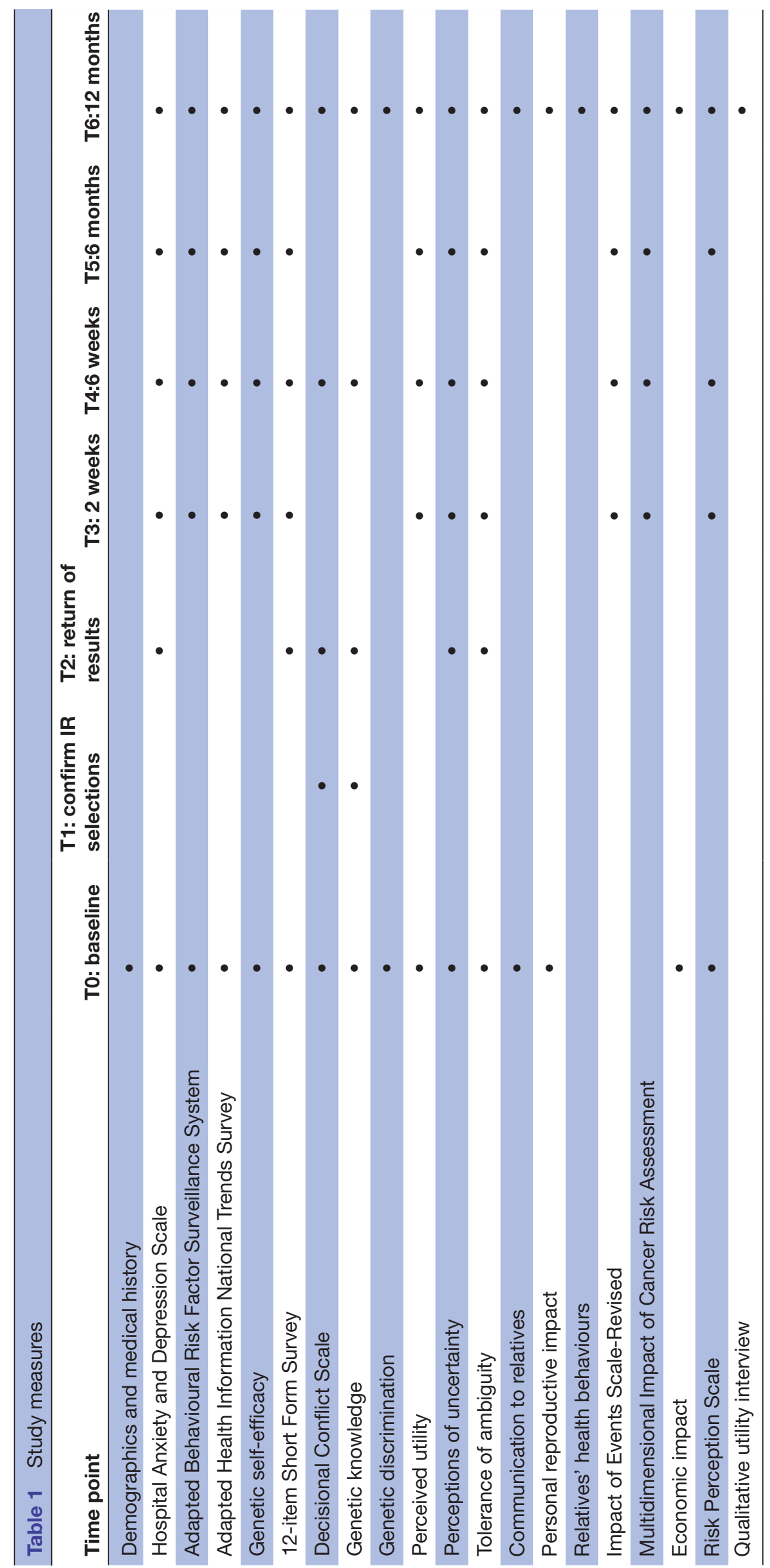

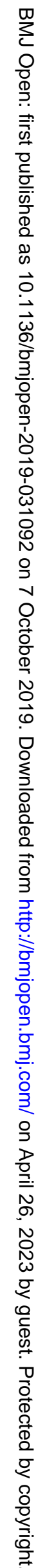


Examples

\section{Category 1: Medically Actionable \\ Category 2: Common Disease Risks \\ Category 3: Rare Mendelian Disorders}

Category 4: Brain Diseases

Category 5: Carrier Status

Figure 2 Categories of incidental results. ${ }^{12}$

\section{Randomisation}

We will consecutively randomise and allocate participants from an existing list of eligible subjects using a computer-generated randomisation in a 1:1 ratio with random permuted blocks of varying sizes, stratified by clinic. Allocations will be generated by a biostatistician and concealed in envelopes by a team member not involved in administering of the study. After a participant is consented and completes baseline measures, the study coordinator will open the envelope and reveal the allocation.

Patients randomised to the intervention arm may not want their IR. This is unlikely given that $>90 \%$ elect to learn at least medically actionable findings. ${ }^{35}$ Nonetheless, patients randomised to the intervention will not have to receive IR; but they will be followed and their outcomes will be included in the intention-to-treat (ITT) analysis as per standard RCT procedures for noncompliance. ${ }^{36}$

\section{Risk of bias}

After assignment of study arm, the research team and participants will not be blinded to allocation, owing to the nature of the intervention. Each participant will be given a code and the data will be analysed blindly. Codes will be accessed after analysis is completed.

\section{Hypotheses, theoretical frameworks, outcomes and analysis}

Aim 1

Evaluate the psychological consequences of receiving IR

\section{Hypothesis}

Compared with patients receiving only primary results, patients receiving IR will report higher levels of distress in the short term (2 weeks), based on genetic testing studies in this population. ${ }^{37}$

\section{Theoretical framework}

Baum's model of stress and genetic testing posits that distress after genetic testing is influenced by disease characteristics (eg, severity, preventability), test result and the extent to which uncertainty is reduced by testing, ${ }^{38}$ suggesting that learning high-risk IR for severe or unpreventable diseases could lead to increased distress. Empirical evidence supports such predictions. The receipt of results from single-gene testing for a range of disorders appears to have adverse psychological effects, including emotional distress for individuals found to be at high risk, but these effects are short lived. ${ }^{39-43}$ Whether such effects are also true for IR from GS, which encompasses results for multiple diseases, is unknown.

\section{Outcomes}

Our primary outcome, psychological distress, will be assessed at 2 weeks, using the validated Hospital Anxiety and Depression Scale (HADS). The HADS is the most commonly used measure of generalised distress in genetic testing. ${ }^{3744}$ The HADS will be assessed at baseline, immediately after results are returned, and 2 weeks, 6 weeks, 6 months and 12 months later (table 1 ).

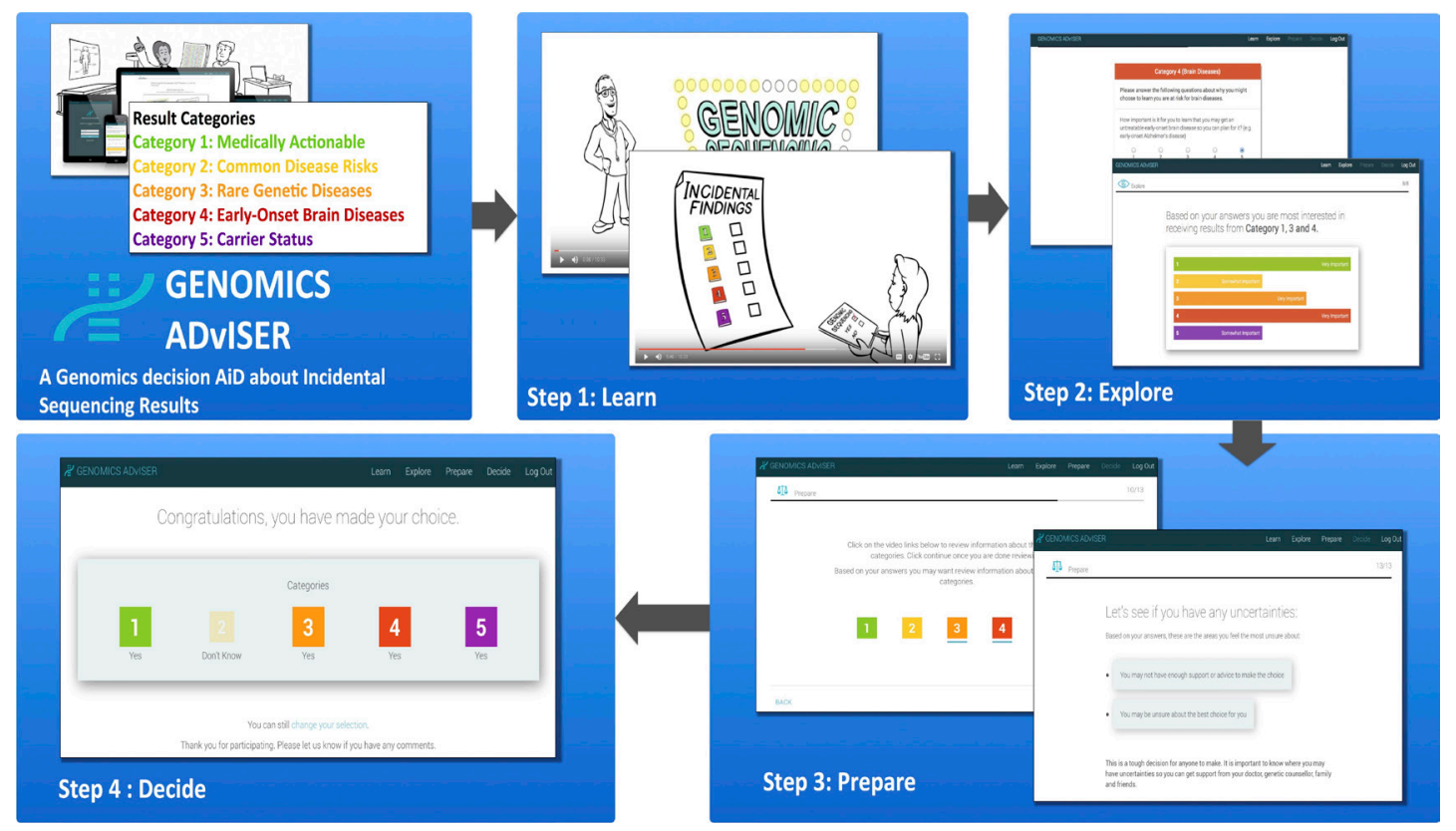

Figure 3 Overview of the GenomicsADvISER.com. ${ }^{12}$ 
Our secondary psychological outcomes are Quality of life measured by the 12-item Short Form Survey (SF-12), ${ }^{45}$ situational distress measured by the Impact of Events Scale Revised (IES-R) ${ }^{46-48}$ and Multidimensional Impact of Cancer Risk Assessment and decisional conflict by the Decisional Conflict Scale, ${ }^{49}$ administered as per the schedule in table 1. Genetic discrimination will also be assessed using an established questionnaire ${ }^{50}$ and GS knowledge will be assessed using a knowledge scale, ${ }^{51}$ administered as per table 1 .

\section{Sample size}

Our primary endpoint is generalised distress using the HADS assessed at 2 weeks. This measure has an accepted minimally important difference of $2.5 .^{52}$ We expect that increased distress will arise from incidental findings, which will only be given in the experimental group. The difference in distress between the groups for those receiving either no findings or only cancer-related findings is expected to be nil. Thus, any difference will be due to the subset of the experimental group that receives incidental findings. This group is expected to comprise $70 \%$ of the experimental group. Therefore, an attenuated treatment effect of $1.75(2.5 \times 0.7)$ on the HADS would be expected when all randomised patients are included. Based on an SD of 5, 130 patients per arm are required to have $80 \%$ power at a two-sided $5 \%$ significance level to detect a treatment effect of a difference of 1.75 on the HADS. $^{52}$

\section{Analysis}

The analysis of distress and secondary outcomes will follow the ITT approach. Our primary time point of the HADS is 2 weeks after return of results. Mean HADS and all baseline measures' scores will be compared at each time point using an analysis of covariance (ANCOVA) model to adjust for baseline values. The sample size presented is for a t-test of the differences. The proposed analysis adjusts for baseline by means of ANCOVA, which has greater power than the t-test, substantially so for moderate to large correlations between baseline and follow-up.

Secondary analyses will adjust for known predictors of the outcome, such as gender and nature of disease/IR. ${ }^{53}$ We will report adjusted mean differences and $95 \%$ CIs of each measure at each follow-up. We will perform a linear mixed-effect model as a secondary analysis to explore if the trajectories differ by group. We will also report the proportion of participants with scores $>11$ on the HADS and $>23$ on the IES-R, indicative of distress at baseline, immediately after return of results and each follow-up.

During each follow-up, we will use a cut-off of $\geq 15$ on the HADS to screen for individuals that may be in acute distress. ${ }^{54}$ The study genetic counsellors will have a discussion with these participants and refer them to a mental health professional or their family doctor if needed.

\section{Aim 2}

Evaluate the behavioural consequences of receiving IR.

\section{Hypothesis}

Compared with patients receiving primary results only, patients receiving IR will report higher levels of intent and uptake for risk-reducing behaviours based on medically actionable and non-actionable results.

We anticipate that most of the IR uncovered by GS will not be medically actionable. However, we expect that these results may still trigger behavioural changes or clinical actions, which will be captured by gathering data from multiple sources, including participants' medical charts, self-reported data and clinical databases.

\section{Theoretical framework}

The Extended Parallel Process Model (EPPM) of health behaviour change suggests that high-risk results (in this case IR) may motivate patients to adopt risk-reducing behaviour, if patients perceive their risk as high and their self-efficacy is also high. ${ }^{55}$ Genetic self-efficacy refers to one's confidence in understanding the role of genetics for health, assessing personal genetic risk for disease and in explaining genetic issues to other individuals. ${ }^{56}$

\section{Outcomes}

We will evaluate intent and uptake of risk-reducing behaviours and preventative services across all RCT participants using modified items from the Centers for Disease Control and Prevention's validated Behavioral Risk Factor Surveillance System (BRFSS) ${ }^{57}$ and the NCI's Health Information National Trends Survey (HINTS). ${ }^{58}$ We have adapted items from the BRFSS and HINTS to examine whether receiving IR influences participants' intention to adopt or their actual self-reported adoption of several risk-reducing behaviours (eg, dietary or medication changes). Consistent with the EPPM model, ${ }^{55}$ we will also assess risk perceptions and genetic self-efficacy using measures adapted from the literature. ${ }^{5659}$

The BRFSS, HINTS, risk perception and self-efficacy measures will be administered at baseline, 2 weeks, 6 weeks, 6 months and 12 months after results are disclosed (table 1). Participants will also be surveyed about intended and actual communication of results to family members and use of results for reproductive decision-making for themselves or their children at each time point using questions derived from the related literature. ${ }^{60}$

Behavioural consequences in relatives: At 6 weeks, 6 months and 12 months, participants will be surveyed about cascade testing, that is, genetic testing of relatives triggered by primary or IR identified in the participant. These data will be triangulated, to the extent possible, by surveying relatives; trial participants will be asked to inform relatives about our interest in following up with them and to provide them with our contact information. If relatives contact us and provide consent, we will assess their intended and actual risk-reducing behaviours using the adapted BRFSS items 6-12 months after results are returned to the trial participant. 


\section{Analysis}

The ITT approach will be followed. We will report the proportion of participants and their relatives reporting intent and adoption of risk-reducing and reproductive behaviours, as attributed to GS results at each time point. The primary time point of interest is 6 months, which will be compared using a $\mathrm{X}^{2}$ test. The absolute risk difference and $95 \%$ CI will be reported. If the data permit, we will explore whether demographic or clinical variables affect behavioural change using multiple logistic regression analysis.

Aim 3

Evaluate the clinical and personal utility of IR.

\section{Quantitative phase}

\section{Hypotheses}

\section{Clinical utility}

1. Medically actionable results: We anticipate that $1 \%-$ $5 \%$ of participants electing to receive medically actionable IR will receive such a result. ${ }^{61}$

2. Non-medically actionable results: For participants electing to receive common disease single-nucleotide polymorphisms (SNPs), carrier status or pharmacogenomics results, we anticipate that $84 \%-100 \%$ will receive such IR. $^{46162}$

3. Medical actions: We anticipate that medically actionable IR will trigger more clinical actions compared with non-medically actionable IR. However, given the higher prevalence of non-medically actionable IR, we anticipate that more individuals will report clinical utility based on the non-medically actionable results compared with medically actionable IR.

\section{Perceived utility}

Compared with patients receiving primary results only, patients receiving IR will report higher levels of perceived utility.

\section{Outcomes}

We will evaluate the clinical utility of IR according to established criteria for evaluating clinical utility of genetic test results. 6364

- Diagnostic yield: We will document the total number of IR, the number and frequency of medically and non-medically actionable results across the five categories of IR and the average number of IR per patient.

- Clinical actions: One year following the return of results, we will record the numbers and types of medical actions recommended and completed following the return of IR obtained through patient chart review.

- Perceived utility: Within our study, we have defined perceived utility as perceived clinical utility and personal utility, which will be evaluated using a scale developed by Lupo et al, ${ }^{65}$ complemented by the qualitative phase per below.

\section{Covariates}

Additional standardised, validated measures of tolerance for ambiguity ${ }^{66}$ and perceptions of uncertainties in GS (PUGS $)^{67}$ will be administered as per the schedule in table 1 . We will also assess clinical variables including: age of cancer diagnosis and family history of cancer (present or absent).

\section{Analysis}

We will report the number of IR and clinical actions across the categories of IR and patients, with 95\% CI when appropriate. We will explore associations between the covariates and perceived utility. The previous literature has indicated that sociodemographic variables are not significant predictors of perceived utility. ${ }^{65}$ Therefore, if the data permit, we will explore whether clinical variables, tolerance of ambiguity and PUGS predict perceived utility of IR using an ordinal regression model.

\section{Qualitative phase}

We will use in-depth interviews with patients and their providers to provide further insight into the ways in which IR may have utility.

Theoretical Framework: Grounded theory ${ }^{68}$ will be used as the qualitative method, given that it is typically used to explore basic social processes based on the theoretical assumptions of symbolic interactionism. ${ }^{69}$

Qualitative Interviews: We will conduct semi-structured interviews to explore clinical utility for clinicians and perceived clinical utility and personal utility for participants of IR 9-12 months after results are returned using interview guides developed based on the literature review and expert consultation. IR may not necessarily trigger clinical actions but still have utility for patients and their relatives. Qualitative interviews will provide insight into outcomes of IR that may not be captured by quantitative measures.

\section{Sample size}

Patients: We will interview up to 40 participants in the intervention arm, a typical sample size for grounded theory studies and usually sufficient to reach thematic saturation. ${ }^{687071} \mathrm{We}$ will first purposively sample across type of IR received (eg, actionable, SNPs, carrier results), and then theoretically sample based on the emerging findings. ${ }^{72} 73$

Providers: With patients' permission, we will interview a subset of specialists $(n=15-20)^{71}$ that intervention patients were referred to as well as a subset of the participants' family physicians $(n=15-20)^{71} 9-12$ months after results are returned. These will constitute a matched sample of patients and their physicians. Fifteen to 20 is a typical sample size for qualitative studies with professionals and sufficient to achieve saturation of themes. $^{687071}$ We will use maximal variation sampling ${ }^{72}$ to ensure data reflect a broad range of practice demographics and across multiple strata, provider experience and GS results. 


\section{Qualitative analysis}

Data analysis will draw on grounded theory methodology. ${ }^{64}$ Qualitative data will be analysed concurrent with data collection to explore preliminary themes and revise the interview guide accordingly. ${ }^{74-78}$ Using HyperRESEARCH, a qualitative data analytical software program, we will sort the data by looking for themes and variations within and across interviews. We will start by open coding the data, which involves labelling the data with descriptive codes. Two team members will code each transcript independently and meet to discuss their analyses until consensus is reached. Inter-rater agreement and kappa coefficients will be calculated to assess intercoder reliability. The next step involves constant comparison, in which codes will be compared across interviews to determine common themes and relationships among them (axial coding). ${ }^{72-74} 77$ The last stage is selective coding, where the codes are integrated to build a theory or explanatory model. Triangulation and member checking will be used as validation methods. $^{78} 79$

\section{Aim 4}

Evaluate the economic consequences of receiving IR.

\section{Hypothesis}

Health service utilisation and consequently health service costs will be higher in patients receiving IR compared with patients receiving primary results only.

\section{Data sources}

We will assess the health service use and costs associated with receiving IR using data from the study and the Institute for Clinical and Evaluative Sciences (IC/ES), an organisation that houses health administrative data for Ontario residents. Each participant will be linked by a unique health insurance number to administrative databases at IC/ES. Patient records will be analysed from IC/ ES at 12 months for short-term analysis (which aligns with the last time point that measures are administered in the RCT) and 5 years after return of results for a longer term analysis.

\section{Health service use}

We will analyse health service use, such as physician visits, emergency room visits, outpatient visits, hospitalisations, medical procedures (eg, surgery, screening), laboratory investigations and additional testing including imaging. We will only consider the services incurred to the health system.

\section{Cost of health services}

We will examine the total cost, and use, by type of service between the two study groups after 12 months and 5 years. The outcome is the incremental cost of the intervention (primary and IR) compared with the control (primary results only) from the perspective of the public health system payer over the study period.
Health service use and economic consequences in relatives

For participating relatives, we will assess health services use and their respective costs using similar methods described above to account for the spillover effects of medical and non-medically actionable IR from GS. For relatives, we also aim to triangulate these data with self-reported data.

\section{Analysis}

Health service use

We will describe the type and frequency of health service use for patients receiving IR versus not receiving IR.

As a secondary analysis, we will explore the potential economic consequences of receiving IR on health service utilisation using a two-part model (a logistic model for the propensity of utilisation and a negative binomial model for the intensity of utilisation). ${ }^{45468081}$ These findings can help explain potential differences in health service use between the two study groups.

\section{Cost of health services}

We will conduct a descriptive analysis to report healthcare costs. Subsequently, we will analyse total cost as a dependent variable, using a regression model, to estimate the difference in expected healthcare costs between the two arms. The intervention variable will be the primary independent variable and the regression model will adjust for potential confounding variables (eg, age, sex and family history) in a generalised linear model. ${ }^{8283}$ We will explore different link functions and family distributions (eg, log link and gamma family) to facilitate careful investigation of the impact that various assumptions have on our conclusions. As secondary outcomes, we will conduct the analysis from the societal perspective where we include all costs from the public health system payer's perspective, including out-of-pocket costs to participants and their relatives using study data. Uncertainty of the findings will be explored and characterised using approaches, such as 95\% CI.

Additionally, if data allow, we will explore the potential cost-effectiveness of IR. For example, we will assess the cost-effectiveness of receiving IR in terms of an incremental cost for one quality-adjusted life year gained. Health-related quality of life data (SF-12) will be collected from the RCT and converted to health utility using a validated algorithm. ${ }^{45}$ Costs will include those described above. We will explore a cost-effectiveness analysis over a 1-year and over a 5-year period to determine whether receiving IR is an overall cost or saving to the public health system.

\section{Recruitment and enrolment}

Figure 4 outlines the recruitment process. Eligible patients will be informed about the study by their genetic counsellor from one of the recruiting clinics. Interested participants will be scheduled for an initial in-person study meeting at $\mathrm{SMH}$ with the study coordinator and study genetic counsellor. 
Retrospective

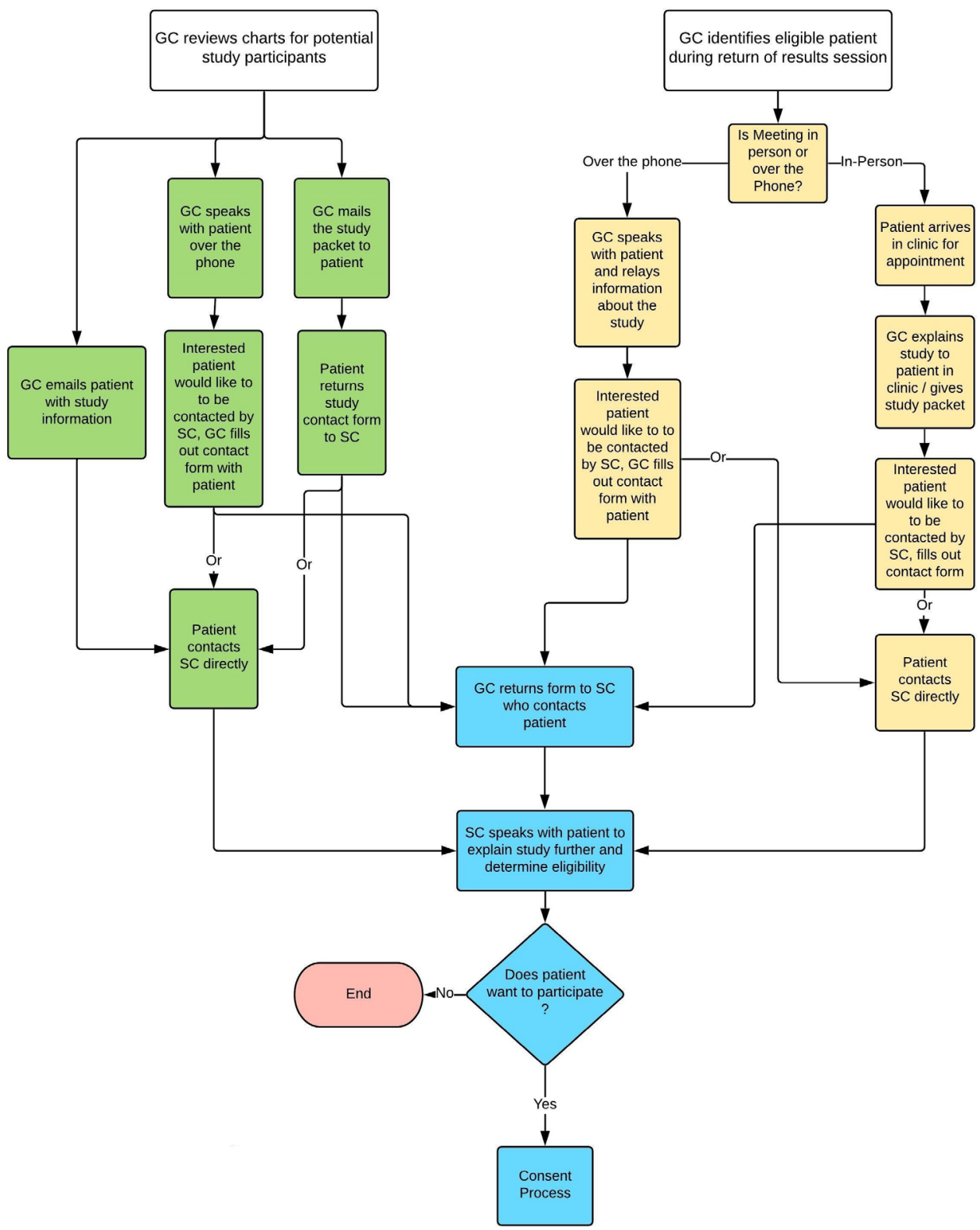

Figure 4 Recruitment process. SC, study coordinator; GC, genetic counsellor

Procedures for qualitative recruitment

A subset of participants who receive IR, their family doctors and specialists will be invited to participate in interviews.

\section{Study flow}

Figure 1 outlines the study procedures. Interested participants will be consented during the in-person meeting and subsequently complete baseline measures, followed by randomisation. Study measures will be collected and managed using Research Electronic Data Capture (REDCap). ${ }^{85}$ The REDCap database will be accessible to approved members of the research team. The server is administered by the Applied Health Research Centre at SMH. Each team member will have their own individual login and password. All data will be kept on secure servers at SMH.
Following randomisation, participants in both arms will receive genetic counselling from a certified genetic counsellor. Participants in the intervention arm will select IR using the DA with additional counselling provided by the study genetic counsellor as needed.

Following the consent session, the participant's exome will be sequenced using DNA from a banked sample or extracted from a fresh blood sample if banked DNA is unavailable.

All DNA samples will be sequenced at The Centre for Applied Genomics (TCAG) in Toronto. TCAG will provide the raw sequencing data for transfer to a secure site at MSH/SHS (Sinai Health System); data will be analysed using a suite of software tools that follow the best practices for analysis of exome data developed by JLE at 
SHS . The study team will assess variants for pathogenicity using established guidelines and categorise results. ${ }^{33}$ Once analysis is complete, reports will be generated by a board certified clinical molecular geneticist JLE (Diplomate of the American Board of Medical Genetics and Genomics, DABMG and Fellow of the American College of Medical Genetics and Genomics, FACMG), Director and Head of the Advanced Molecular Diagnostic Laboratory (AMDL) or JCC (FACMG) at MSH and the analysis team.

Once the reports are generated, the study counsellor will consult with RHK, the clinical geneticist for the RCT, about appropriate actions for the primary and IR. The study genetic counsellor will relay this information to the recruiting genetic counsellor and together, they will devise a plan for return of results.

Following the results session, the study genetic counsellor, in consultation with JLE and RHK, will develop consult notes summarising the results, recommendations and suggested referrals for both the primary results and IR. The consult note will be forwarded to the recruiting clinic that will facilitate the referrals to the appropriate genetics clinic for clinical confirmation and counselling. An expedited system has been set up with local genetics clinics to facilitate appointments for participants with positive results from the trial. Each participant and their family physician will receive a consultation note (online supplementary files 2,3). After the results session, follow-up measures will be evaluated over the phone with an SMH study coordinator per the schedule in table 1 . If participants miss any sessions, we will follow up with them to ensure they complete their measures on time. For participants who drop out of the study, we will still have access to their medical records and be able to evaluate their health outcomes.

We also will send each participant's family physician a letter notifying them of their patient's enrolment (online supplementary file 4).

Participants can choose to leave the study at any time. If participants leave prior to sequencing of their DNA, their sample will be destroyed. For participants who leave after sequencing, the raw sequence data will be destroyed, unless it has already been uploaded to a database. In this case, the data will not be removable from the database, but participants can choose to remove their data from the study itself. Results of any analysis, including sequencing data and any other information recorded before withdrawal, will still be used by the researchers for the study purposes, but no new information will be collected.

Any providers who agree to interviews will be consented by the study coordinator and interviewed by phone.

\section{Genomic sequencing and binning framework}

GS will be performed using next-generation sequencing on the Illumina HiSeq 2500 platform. The raw sequence reads will be analysed at secure sites at $\mathrm{SMH}$ and $\mathrm{MSH}$ using AMDL's GS pipeline that uses alternative and validated software tools that have been cross-validated against internal standards.
For participants in the control group, raw sequencing data will be filtered to exclude variants not associated with cancer, so that the laboratory and research team are blinded to non-cancer results. Therefore, variants for IR will not be analysed until the 1-year time point for these participants. In the case that a medically actionable IR is inadvertently identified for a control participant in the analysis process, the result will be returned to the participant immediately and will not be withheld until the end of the study.

For the primary cancer indication, a list of 151 genes was generated using genes from hereditary cancer panels issued by reputable labs, Invitae, GeneDx, Ambry Genetics and the Catalogue of Somatic Mutations in Cancer v86 database.

Intervention participants will select IR using a modified version of Berg's proposed binning structure, described above $^{32}$ (figure 2). The development of our binning structure and list are elaborated elsewhere. ${ }^{12}{ }^{86}$ Briefly, Bin 1 encompasses medically actionable genes and was developed using the 59 genes recommended by the ACMG and The Clinical Genome Resource's list of actionable genes. ${ }^{387}$ Currently, the list contains 90 genes. Bin 1 also includes pharmacogenomic variants, extracted from the Pharmacogenomics Knowledgebase, a publicly available interactive database of pharmacogenomics variants. Bin 2 is composed of SNPs for common diseases (eg, age-related macular degeneration). SNPs are variations in a single position in the DNA sequence between individuals that can be associated with small increased or decreased risks of developing diseases. SNPs that reached genomewide significance $\left(\mathrm{p}<5 \times 10^{-8}\right)$ and had a reported $\mathrm{OR} \geq 2.0$ from the NHGRI-EBI Catalogue (V.1.0.2) of published genome-wide association studies were included. Bin 3 is composed of all genes associated with Mendelian disorders not included in Bin 1 or Bin 4; the list was generated using Online Mendelian Inheritance in Man. ${ }^{88}$ Bin 4 is composed of early-onset neurological disorders, such as early-onset familial Alzheimer's disease ${ }^{89-91}$ Bin 5, carrier results, was developed using genes used by the Clinical Sequencing Exploratory Research consortium. ${ }^{92}$

\section{Patient and public involvement}

We have recently formed a patient advisory board that we will engage over the course of our trial. These patients will be involved with the conduct and reporting of the study.

\section{ETHICS AND DISSEMINATION \\ Ethics}

In the case that protocol amendments are required, revisions will be submitted to the Streamlined Research Ethics Review System. All changes will be communicated to the study team and ethics board. If there are any changes that directly affect patients or require consent, all enrolled patients will be informed of the changes. All participants will be consented in person by the study coordinator, who will review the form in detail, answer questions and obtain permission to access medical chart information. Participants will receive a copy of the form 
(online supplementary file 5). All information collected will be kept confidential. There will be data and sample transfer agreements between SMH and each site. The study will not have a data monitoring committee given that we do not anticipate severe adverse effects and was not required for our study by the Research Ethics Board (REB). To assure the conduct of quality research, the St. Michael's Hospital Research Ethics Board does regularly conduct audits of research studies.

\section{Dissemination}

We intend to share results through local, national and international conferences and publications in peer-reviewed journals. Authorship eligibility will be based on The International Committee of Medical Journal Editors. Furthermore, we will organise a stakeholder workshop with genetic counsellors, geneticists, oncologists, family physicians, laboratory professionals and patients to develop recommendations to optimise the return of IR from GS in clinical practice. Our team will also work with GEC-KO (Genetics Education Canada) (http://geneticseducation.ca), of which JCC is a codirector, in order to develop educational materials on managing IR for primary care physicians, based on study results. Finally, we will develop policy briefs in collaboration with our policy partners from Health Quality Ontario and the Canadian Agency for Drugs and Technology in Health to inform health technology assessment and funding decisions for GS.

The final trial dataset will be accessed by the principal investigator, immediate study team and biostatistician.

\section{Author affiliations}

${ }^{1}$ Institute of Health Policy, Management and Evaluation, University of Toronto, Toronto, Ontario, Canada

${ }^{2}$ Genomics Health Services Research Program, Li Ka Shing Knowledge Institute, St. Michael's Hospital, Toronto, Ontario, Canada

${ }^{3}$ Lunenfeld-Tanenbaum Research Institute, Mount Sinai Hospital, Sinai Health

System, Toronto, Ontario, Canada

${ }^{4}$ Faculty of Medicine, University of Toronto, Toronto, Ontario, Canada

${ }^{5}$ Marvelle Koffler Breast Centre, Mount Sinai Hospital, Sinai Health System, Toronto, Ontario, Canada

${ }^{6}$ Odette Cancer Centre, Sunnybrook Health Sciences Centre, Toronto, Ontario,

Canada

${ }^{7}$ Zane Cohen Centre for Digestive Diseases, Mount Sinai Hospital, Sinai Health System, Toronto, Ontario, Canada

${ }^{8}$ Familial Breast Ovarian Cancer Clinic, Princess Margaret Cancer Centre, Toronto, Ontario, Canada

${ }^{9}$ Fred A. Litwin Centre in Genetic Medicine, University Health Network, Toronto, Ontario, Canada

${ }^{10}$ Department of Laboratory Medicine, St. Michael's Hospital, Toronto, Ontario, Canada

${ }^{11}$ Department of Laboratory Medicine and Pathobiology, University of Toronto, Toronto, Ontario, Canada

${ }^{12}$ GeneDx, Elmwood Park, New Jersey, USA

${ }^{13}$ Department of Molecular Oncology and Hereditary Cancer Program, BC Cancer

Agency, Vancouver, British Columbia, Canada

${ }^{14}$ Department of Medical Genetics, The University of British Columbia, Vancouver, British Columbia, Canada

${ }^{15}$ Clinical Genetics Service, Department of Medicine, Memorial Sloan Kettering Cancer Center, New York City, New York, USA

${ }^{16}$ Breast Medicine, Memorial Sloan Kettering Cancer Center, New York City, New York, USA
${ }^{17}$ Ray D Wolfe Department of Family Medicine, Mount Sinai Hospital, Sinai Health System, Toronto, Ontario, Canada

${ }^{18}$ Department of Family and Community Medicine, University of Toronto, Toronto,

Ontario, Canada

${ }^{19}$ Centre for exceLlence in Economic Analysis Research (CLEAR), Li Ka Shing Knowledge Institute, St. Michael's Hospital, Toronto, Ontario, Canada

${ }^{20}$ Division of Medical Oncology and Hematology, University Health Network, Toronto, Ontario, Canada

${ }^{21}$ Applied Health Research Centre, Li Ka Shing Knowledge Institute, St. Michael's Hospital, Toronto, Ontario, Canada

${ }^{22}$ Dalla Lana School of Public Health, University of Toronto, Toronto, Ontario, Canada

${ }^{23}$ Department of Palliative Care, St. Michael's Hospital, Toronto, Ontario, Canada

Acknowledgements We would like to thank the following individuals for supporting this study: Carolyn Piccinin, Laura Winter-Paquette, Kara Semotiuk, Talia Mancuso, Justin Lorentz, Karen Ott, Yael Silberman, Rochelle Demsky, Alexandra Volenik and Jeanna McCuaig.

Collaborators Incidental Genomics Study Team: Yvonne Bombard (PI) ; Susan Randall Armel; Melyssa Aronson; Nancy Baxter; Ken Bond; José-Mario Capo-Chichi; June C. Carroll; Timothy Caulfield; Marc Clausen; Tammy J. Clifford; Iris Cohn; Irfan Dhalla; Craig C. Earle; Andrea Eisen; Christine Elser; Mike Evans; Emily Glogowski; Tracy Graham; Jada G. Hamilton; Wanrudee Isaranuwatchai; Monika Kastner; Raymond H. Kim; Andreas Laupacis; Jordan Lerner-Ellis; Chantal F. Morel; Michelle Mujoomdar; Kenneth Offit; Seema Panchal; Mark Robson; Stephen W. Scherer; Adena Scheer; Kasmintan A. Schrader; Terrence Sullivan and Kevin E. Thorpe.

Contributors YB led the study, conceived and designed the protocol. YB, KET, AL and WI codesigned the protocol. MC codesigned the study and wrote the protocol. Members of the Incidental Genomics Study Team including CFM, EG, KAS, JGH, KO, MR, JCC, RHK, JL-E and RK, ER, SS, MGS, CM, K-RZ and RF informed the design of the protocol. MC, SS, CM, RK, ER, CE, SP, AE, TG, MA and SRA will assist in data collection. SS, MC and CM assisted with drafting the manuscript. All authors read and approved the final manuscript

Funding statement This study was supported by a Foundation Grant from the Canadian Institutes of Health Research and a Quality of Life Grant from the Canadian Cancer Society Research Institute awarded to YB (grant numbers 143310 and 705665 , respectively). YB was supported by a New Investigator Award from the Canadian Institute of Health Research during the conduct of this study. JGH, KO and MR were supported by NCI P30 CA008748.

Disclaimer The funders have no role in study design, data collection and analysis, decision to publish or preparation of manuscripts.

Competing interests None declared.

Patient consent for publication Not required.

Ethics approval Clinical Trials Ontario Streamlined Research Ethics Review System (SRERS) has approved the study at participating sites.

Provenance and peer review Not commissioned; externally peer reviewed.

Open access This is an open access article distributed in accordance with the Creative Commons Attribution Non Commercial (CC BY-NC 4.0) license, which permits others to distribute, remix, adapt, build upon this work non-commercially, and license their derivative works on different terms, provided the original work is properly cited, appropriate credit is given, any changes made indicated, and the use is non-commercial. See: http://creativecommons.org/licenses/by-nc/4.0/.

\section{REFERENCES}

1 Schwarze K, Buchanan J, Taylor JC, et al. Are whole-exome and whole-genome sequencing approaches cost-effective? A systematic review of the literature. Genet Med 2018;20:1122-30.

2 National Human Genome Research Institute. The cost of sequencing a human genome; 2016. https://www.genome.gov/about-genomics/ fact-sheets/Sequencing-Human-Genome-cost

3 Kalia SS, Adelman K, Bale SJ, et al. Recommendations for reporting of secondary findings in clinical exome and genome sequencing, 2016 update (ACMG SF v2.0): a policy statement of the American College of medical genetics and genomics. Genet Med 2017:19:249-55.

4 Amendola LM, Dorschner MO, Robertson PD, et al. Actionable exomic incidental findings in 6503 participants: challenges of variant classification. Genome Res 2015;25:305-15. 
5 Jarvik GP, Amendola LM, Berg JS, et al. Return of genomic results to research participants: the floor, the ceiling, and the choices in between. Am J Hum Genet 2014;94:818-26.

6 van El CG, Cornel MC, Borry P, et al. Whole-Genome sequencing in health care: recommendations of the European Society of human genetics. Eur J Hum Genet 2013;21:580-4.

7 Boycott K, Hartley T, Adam S, et al. The clinical application of genome-wide sequencing for monogenic diseases in Canada: position statement of the Canadian College of medical geneticists. $J$ Med Genet 2015;52:431-7.

8 Hehir-Kwa JY, Claustres M, Hastings RJ, et al. Towards a European consensus for reporting incidental findings during clinical NGS testing. Eur J Hum Genet 2015;23:1601-6.

9 Cambridge UK. Managing incidental and pertinent findings from WGS in the 100,000 genomes project, PHG Foundation, 2013. ISBN: 978-1-907198-12-0.

10 Shahmirzadi L, Chao EC, Palmaer E, et al. Patient decisions for disclosure of secondary findings among the first 200 individuals undergoing clinical diagnostic exome sequencing. Genet Med 2014:16:395-9.

11 Regier DA, Peacock SJ, Pataky R, et al. Societal preferences for the return of incidental findings from clinical genomic sequencing: a discrete-choice experiment. Can Med Assoc J 2015;187:E190-E197.

12 Bombard Y, Clausen M, Mighton C, et al. The genomics ADVISER: development and usability testing of a decision aid for the selection of incidental sequencing results. Eur J Hum Genet 2018;26:984-95.

13 Facio $\mathrm{FM}$, Eidem $\mathrm{H}$, Fisher $\mathrm{T}$, et al. Intentions to receive individual results from whole-genome sequencing among participants in the ClinSeq study. Eur J Hum Genet 2013;21:261-5.

14 Daack-Hirsch S, Driessnack M, Hanish A, et al. 'Information is information': a public perspective on incidental findings in clinical and research genome-based testing. Clin Genet 2013;84:11-18.

15 Parsons DW, Roy A, Yang Y, et al. Diagnostic yield of clinical tumor and germline whole-exome sequencing for children with solid tumors. JAMA Oncol 2016;2.

16 Shoemaker JM, Cole C, Petree LE, et al. Evolution of universal review and disclosure of MRI reports to research participants. Brain Behav 2016;6:e00428.

17 Phillips JP, Cole C, Gluck JP, et al. Stakeholder opinions and ethical perspectives support complete disclosure of incidental findings in MRI research. Ethics Behav 2015;25:332-50.

18 Kohane IS, Taylor PL. Multidimensional results reporting to participants in genomic studies: getting it right. Sci Trans/ Med 2010;2:37cm19.

19 Lázaro-Muñoz G, Conley JM, Davis AM, et al. Which results to return: subjective judgments in selecting medically actionable genes. Genet Test Mol Biomarkers 2017:21:184-94.

20 Hunter JE, Irving SA, Biesecker LG, et al. A standardized, evidencebased protocol to assess clinical actionability of genetic disorders associated with genomic variation. Genet Med 2016;18:1258-68.

21 Ormondroyd E, Mackley MP, Blair E, et al. "Not pathogenic until proven otherwise": perspectives of UK clinical genomics professionals toward secondary findings in context of a Genomic Medicine Multidisciplinary Team and the 100,000 Genomes Project. Genet Med 2018;20:320-8.

22 Barwell JG, O'Sullivan RBG, Mansbridge LK, et al. Challenges in implementing genomic medicine: the 100,000 genomes project. $J$ Transl Genet Genom 2018;2.

23 Chan A-W, Tetzlaff JM, Altman DG, et al. Spirit 2013 statement: defining standard protocol items for clinical trials. Ann Intern Med 2013;158:200-7.

24 Chan A-W, Tetzlaff JM, Gøtzsche PC, et al. Spirit 2013 explanation and elaboration: guidance for protocols of clinical trials. BMJ 2013;346:e7586.

25 Kalia M. Biomarkers for personalized oncology: recent advances and future challenges. Metabolism 2015;64(3 Suppl 1):S16-S21.

26 Bombard Y, Bach PB, Offit K. Translating genomics in cancer care. J Natl Compr Canc Netw 2013;11:1343-53.

27 Hyman DM, Solit DB, Arcila ME, et al. Precision medicine at Memorial Sloan Kettering cancer center: clinical next-generation sequencing enabling next-generation targeted therapy trials. Drug Discov Today 2015;20:1422-8.

28 Stavropoulos DJ, Merico D, Jobling R, et al. Whole-Genome sequencing expands diagnostic utility and improves clinical management in paediatric medicine. NPJ Genom Med 2016;1.

29 Miller FA, Hayeems RZ, Bytautas JP, et al. Testing personalized medicine: patient and physician expectations of next-generation genomic sequencing in late-stage cancer care. Eur J Hum Genet 2014;22:391-5.

30 Hamilton JG, Shuk E, Genoff MC, et al. Interest and attitudes of patients with advanced cancer with regard to secondary germline findings from tumor genomic profiling. $J$ Oncol Pract 2017;13:e590-601.

31 Shickh S, Clausen M, Mighton C, et al. Evaluation of a decision aid for incidental genomic results, the Genomics ADVISER: protocol for a mixed methods randomised controlled trial. BMJ Open 2018;8:e021876.

32 Berg JS, Khoury MJ, Evans JP. Deploying whole genome sequencing in clinical practice and public health: meeting the challenge one bin at a time. Genet Med 2011;13:499-504.

33 Richards S, Aziz N, Bale S, et al. Standards and guidelines for the interpretation of sequence variants: a joint consensus recommendation of the American College of Medical Genetics and Genomics and the Association for Molecular Pathology. Genet Med 2015;17:405-23.

34 Bombard Y, Brothers KB, Fitzgerald-Butt S, et al. The responsibility to recontact research participants after reinterpretation of genetic and genomic research results. Am J Hum Genet 2019;104:578-95.

35 Loud JT, Bremer RC, Mai PL, Peters JA, et al. Research participant interest in primary, secondary, and incidental genomic findings. Genet Med 2016;18:1218-25.

36 Gupta SK. Intention-To-Treat concept: a review. Perspect Clin Res 2011;2:109-12.

37 Hamilton JG, Lobel M, Moyer A. Emotional distress following genetic testing for hereditary breast and ovarian cancer: a meta-analytic review. Health Psychol 2009;28:510-8.

38 Baum A, Friedman AL, Zakowski SG. Stress and genetic testing for disease risk. Health Psychol 1997;16:8-19.

39 Meiser B, Collins V, Warren R, et al. Psychological impact of genetic testing for hereditary non-polyposis colorectal cancer. Clin Genet 2004;66:502-11. CGE339 [pii].

40 Watson M, Foster C, Eeles R, et al. Psychosocial impact of breast/ ovarian (BRCA1/2) cancer-predictive genetic testing in a UK multicentre clinical cohort. Br J Cancer 2004;91:1787-94

41 Green RC, Roberts JS, Cupples LA, et al. Disclosure of APOE genotype for risk of Alzheimer's disease. $N$ Engl J Med 2009;361:245-54. 361/3/245 [pii].

42 Lerman C, Narod S, Schulman K, et al. BRCA1 testing in families with hereditary breast-ovarian cancer. A prospective study of patient decision making and outcomes. JAMA 1996;275:1885-92.

43 Arver B, Haegermark A, Platten U, et al. Evaluation of psychosocial effects of pre-symptomatic testing for breast/ovarian and colon cancer pre-disposing genes: a 12-month follow-up. Fam Cancer 2004;3:109-16.

44 Payne K, Nicholls S, McAllister M, et al. Outcome measurement in clinical genetics services: a systematic review of validated measures. Value Health 2008;11:497-508.

45 Johnson JA, Coons SJ. Comparison of the EQ-5D and SF-12 in an adult US sample. Qual Life Res 1998;7:155-66.

46 Horowitz M, Wilner N, Alvarez W. Impact of event scale: a measure of subjective stress. Psychosom Med 1979;41:209-18.

47 Zilberg NJ, Weiss DS, Horowitz MJ. Impact of event scale: a cross-validation study and some empirical evidence supporting a conceptual model of stress response syndromes. J Consult Clin Psychol 1982;50:407-14.

48 Creamer M, Bell R, Failla S. Psychometric properties of the Impact of Event Scale - Revised. Behav Res Ther 2003;41:1489-96.

49 O'Connor AM. Validation of a decisional conflict scale. Med Decis Making 1995;15:25-30.

50 Bombard Y, Veenstra G, Friedman JM, et al. Perceptions of genetic discrimination among people at risk for Huntington's disease: a cross sectional survey. BMJ 2009;338:b2175.

51 Kaphingst KA, Facio FM, Cheng M-R, et al. Effects of informed consent for individual genome sequencing on relevant knowledge. Clin Genet 2012;82:408-15.

52 Chan KS, Aronson Friedman L, Bienvenu OJ, et al. Distributionbased estimates of minimal important difference for hospital anxiety and depression scale and impact of event scale-revised in survivors of acute respiratory failure. Gen Hosp Psychiatry 2016;42:32-5.

53 Strong V, Waters R, Hibberd C, et al. Emotional distress in cancer patients: the Edinburgh cancer centre symptom study. $\mathrm{Br} \mathrm{J}$ Cancer 2007;96:868-74.

54 Walker J, Postma K, McHugh GS, et al. Performance of the hospital anxiety and depression scale as a screening tool for major depressive disorder in cancer patients. J Psychosom Res 2007;63:83-91.

55 Witte K, Cameron KA, McKeon JK, et al. Predicting risk behaviors: development and validation of a diagnostic scale. J Health Commun 1996;1:317-42.

56 Kaphingst KA, McBride CM, Wade C, et al. Patients' understanding of and responses to multiplex genetic susceptibility test results. Genet Med 2012;14:681-7. 
57 Mokdad $\mathrm{AH}$. The behavioral risk factors surveillance system: past, present, and future. Annu Rev Public Health 2009;30:43-54.

58 Health Information National Trends Survey. U.S. department of health and human services; 2018. https://hints.cancer.gov/docs/ Instruments/HINTS5_Cycle1_Annotated_Instrument_English.pdf.

59 Cameron LD, Sherman KA, Marteau TM, et al. Impact of genetic risk information and type of disease on perceived risk, anticipated affect, and expected consequences of genetic tests. Health Psychol 2009;28:307-16.

60 Bombard Y, Miller FA, Barg CJ, et al. A secondary benefit: the reproductive impact of carrier results from newborn screening for cystic fibrosis. Genet Med 2017;19:403-11.

61 Gambin T, Jhangiani SN, Below JE, et al. Secondary findings and carrier test frequencies in a large multiethnic sample. Genome Med 2015;7:54.

62 Dorschner MO, Amendola LM, Turner EH, et al. Actionable, pathogenic incidental findings in 1,000 participants' exomes. Am J Hum Genet 2013:93:631-40.

63 Sun F, Bruening W, Erinoff E, et al. Addressing challenges in genetic test evaluation: evaluation frameworks and assessment of analytic validity. Agency for Healthcare Research and Quality 2011.

64 Teutsch SM, Bradley LA, Palomaki GE, et al. The evaluation of genomic applications in practice and prevention (EGAPP) initiative: methods of the EGAPP Working group. Genet Med 2009;11:3-14.

65 Lupo PJ, Robinson JO, Diamond PM, et al. Patients' perceived utility of whole-genome sequencing for their healthcare: findings from the MedSeq project. Per Med 2016;13:13-20.

66 Geller G, Tambor ES, Chase GA, et al. Measuring physicians' tolerance for ambiguity and its relationship to their reported practices regarding genetic testing. Med Care 1993;31:989-1001.

67 Biesecker BB, Woolford SW, Klein WMP, et al. PUGS: a novel scale to assess perceptions of uncertainties in genome sequencing. Clin Genet 2017;92:172-9.

68 Strauss A, Corbin JM. Basics of qualitative research: techniques and procedures for developing grounded theory. Second ed. CA: Sage Publications, 1998.

69 Blumer H. Symbolic interactionism; perspective and method. Englewood Cliffs, N.J.: Prentice-Hall, 1969.

70 Morse J. Designing funded qualitative research. In: Denzin NKL, ed. Handbook of qualitative research. thousand oaks, Ca: SAGE publications. Y.S, 1994: 220-35.

71 Sandelowski M. Sample size in qualitative research. Res Nurs Health 1995;18:179-83.

72 Coyne IT. Sampling in qualitative research. purposeful and theoretical sampling; merging or clear boundaries? J Adv Nurs 1997;26:623-30.

73 Miles M, Huberman M. Qualitative data analysis; an expanded sourcebook. Newbury Park, CA: Sage Publications, 1994.
74 Charmaz KC. Constructing Grounded theory: a practical guide through qualitative analysis. London: SAGE Publications Ltd, 2006.

75 Strauss AL, Corbin J. Basics of qualitative research: Grounded theory procedures and techniques. Newbury Park, CA: Sage, 1990.

76 Charmaz KC. Grounded theory: Objectivist and constructivist methods. In: Denzin NK, Lincoln YS, Oaks T, eds. Handbook of qualitative research. 2nd ed. CA: Sage, 2000: 509-35.

77 Charmaz KC. Qualitative interviewing and grounded theory analysis. In: Holstein JA, Gubrium JF, Oaks T, eds. Inside interviewing: new lenses, new concerns. CA: Sage Publications Inc, 2003: 311-30.

78 Morse JM. Approaches to qualitative-quantitative methodological triangulation. Nurs Res 1991;40:120-3.

79 Sandelowski M. Rigor or rigor mortis: the problem of rigor in qualitative research revisited. ANS Adv Nurs Sci 1993;16:1-8.

80 Deb P, Trivedi PK. The structure of demand for health care: latent class versus two-part models. $J$ Health Econ 2002;21:601-25.

81 Belotti F, Deb P, Manning WG, et al. Twopm: two-part models. Stata J 2015;15:3-20.

82 Briggs A, Nixon R, Dixon S, et al. Parametric modelling of cost data: some simulation evidence. Health Econ 2005;14:421-8.

83 Barber J, Thompson S. Multiple regression of cost data: use of generalised linear models. J Health Serv Res Policy 2004;9:197-204

84 Measuring and valuing health, 2018. The University of Sheffield United Kingdom. Available: https://www.sheffield.ac.uk/scharr/ sections/heds/mvh/sf-6d.

85 REDCap. Research electronic data capture; 2019. https://redcap. smh.ca/redcap/2019

86 Gutierrez Salazar Met al. An updated binning framework for the return of secondary findings in diagnostic whole exome sequencing, in preparation.

87 ClinGen.The Clinical Genome Resource., 2018. Available: https:// actionability.clinicalgenome.org/site/cg-actionability

88 OMIM. Online Mendelian Inheritance in Man, 2018. Available: https:// www.omim.org/.

89 Bird T. Early onset familial Alzheimer disease, 2018. Available: https:// www.ncbi.nlm.nih.gov/books/NBK1236/

90 Puschmann A. Monogenic Parkinson's disease and parkinsonism: clinical phenotypes and frequencies of known mutations. Parkinsonism Relat Disord 2013;19:407-15.

91 Sundal C, Fujioka S, Van Gerpen JA, et al. Parkinsonian features in hereditary diffuse leukoencephalopathy with spheroids (HDLS) and CSF1R mutations. Parkinsonism Relat Disord 2013;19:869-77.

92 Himes P, Kauffman TL, Muessig KR, et al. Genome sequencing and carrier testing: decisions on categorization and whether to disclose results of carrier testing. Genet Med 2017;19:803-8. 